INGRAM, G. I. C. (1951). J. gen. Microbiol. 5, 22-29.

\title{
Formation of Glear Zones with 'Sensitive' and 'Resistant' Staphylococcus aureus in Penicillin Plate Assays
}

\author{
BY G. I. C. INGRAM \\ Department of Pathology, St Thomas's Hospital, Hydestile, Surrey
}

SUMMARY: The high-power microscopical examination of stained specimens of portions of agar plate cultures of bacteria allows bacterial cells and colonies to be studied in their original positions. The disposition and morphology of bacteria from the edges of clear zones on penicillin assay plates under conditions sufficiently standardized permitted a distinction between sensitive and resistant strains of Staphylococcus aureus. With a sensitive strain 'ghost zones' were formed of partially lysed, distorted and abnormally staining cells; with a resistant strain a 'scatter zone' was formed of apparently normal cells in isolated colonies.

For plate assay work, a sensitive strain may be used when only low concentrations of the drug are available; a resistant strain may be expected to give a sharper zone edge, but will require higher concentrations to provide an adequate response.

Examinations of assay plates have been made by a number of workers in studying the antibacterial properties of penicillin. The wide use of Staph. aureus for penicillin assay has prompted the inclusion of penicillin-sensitive strains in such investigations, but a detailed comparative study of assay plates seeded with sensitive and resistant forms does not appear to have been reported.

From direct examination of assay plates, numerous observations on the structure of inhibition zones have been reported. Abraham, Chain, Fletcher, Gardner, Heatley, Jennings \& Florey (1941) mentioned that indefinite zones might occur between the clear circle and the opaque normal growth in the cylinder-plate method. Foster \& Woodruff (1943) noticed an indistinctness of the clear-zone edge, attributed to lysis of the staphylococci; the variable nature of intermediate zones was also noted. Knox (1945) used a $5 \%$ horseblood agar medium and a single central cylinder of penicillin solution to each Petri dish, multiple inocula being made in radial streaks. With sensitive staphylococci examination under $10 \times$ magnification confirmed the naked-eye impression that lysis of colonies occurred in the periphery of the cleared part of the streaks. Haemolysis in the region of bacteriolysis suggested that bacterial growth here had previously been vigorous, although only the debris of colonies remained at the end of incubation. With a resistant staphylococcus, lysis was not seen, and the zone of haemolysis did not extend inwards from the region of normal growth.

Vesterdal (1946), using an all-over surface-seeding technique with a sensitive staphylococcus, also noted the extension of clear-zone edges by lysis of marginal colonies, and recorded the diameters of outer and intermediate zone edges. With the lowest concentrations of penicillin used the intermediate zones were narrow and opaque. With greater concentrations these zones widened, and an inner, translucent zone appeared, which gradually displaced the opaque zone, and became still wider as the series was continued to the highest concentrations 
tested. Vesterdal related the opaque intermediate zone to the development of resistant colonies, made possible by a slower diffusion of penicillin from reservoirs of low concentration, whereas the transparent zone was attributed to lysis. Pulvertaft, Greening \& Haynes (1947) found that various combinations of strains and media produced constant patterns of rings with each batch of agar, differing widely between different batches.

Two other lines of study contributed to an understanding of phenomena observed on assay plates: bacterial growth studied in fluid media containing various concentrations of penicillin, and the microscopical observation of organisms while growing in the presence of the drug. The outstanding early fluid culture studies were those of Chain, Duthie \& Callow (1945), Kirby $(1945 a, b)$, and Todd (1945) based on nephelometry, and supplemented in the first instance by viable counts and haemocytometer cell counts. Early studies with the incubating microscope were reported by Gardner (1940, 1945), Duguid (1946) and Eriksen (1946). From this work it appears that penicillinsensitive staphylococci begin to grow normally in low penicillin concentrations, but undergo a gross distortion and lysis when the phase of logarithmic division is reached. It is reported incidentally that the penicillin-resistant type of staphylococcus may not show lysis.

\section{EXPERIMENTAL}

In the present work, comparisons were made between the structure of clearzone edges on assay plates seeded with sensitive and with resistant staphylococci, paying particular attention to penicillinase formation, inoculum size and the occurrence of lysis and morphological change due to penicillin activity.

\section{Method for direct examination of organisms on agar plates}

Since direct microscopical examination of the surface of assay plates with a $\frac{1}{12}$ in. oil immersion objective proved unsatisfactory, the following procedure was adopted for examining thin stained slips of agar from the surface of the plate without disturbing the arrangement of the bacteria.

(For the purpose of the following discussion a Petri plate consists of a lower, smaller part, the dish, and an upper, larger part, the lid.)

Flat-bottomed Petri dishes, preferably $15 \mathrm{~cm}$. in diameter, were levelled and poured to a depth of about $5 \mathrm{~mm}$. with ordinary $2 \%$ nutrient agar medium containing 5-10\% of $\mathrm{NaCl}$ to suppress air contaminants (Chapman, 1945). When the medium had solidified, the plate was warmed in the $37^{\circ}$ incubator and covered with a layer $0.5-1.0 \mathrm{~mm}$. thick of the same nutrient agar diluted with an equal volume of $\mathrm{NaCl}$ broth and seeded with the staphylococcus. A suitable density of seeding is usually attained by adding about $0.1 \mathrm{ml}$. of an overnight broth culture of the organism to $10 \mathrm{ml}$. of the diluted agar after melting and allowing it to cool to $40^{\circ}$.

When the second layer of agar had set, the preparation was dried in the incubator for $30 \mathrm{~min}$., with the lid raised. Blotting-paper disks carrying the penicillin solution were then laid on the pre-seeded surface (disks of any diameter between 10 and $2 \mathrm{~mm}$. may be used) and the plate closed. After 
incubation (not inverted), the lid was raised again and a further period of about an hour allowed in the incubator, to remove free surface moisture. This important step prevented the most superficial portion of the growth from washing to and fro during manipulation.

The plate was then inverted on the bench and 1-2 ml. of Liq. Formaldehyd B.P. (c. $39 \%$ $(\mathrm{w} / \mathrm{v}) \mathrm{HCHO})$ placed in the lid, and the preparation allowed to stand for about $3 \mathrm{hr}$. It may be left until the following day provided further loss of water is prevented.

Colony dispositions could then be examined directly with the $\frac{2}{3}$ in. objective.

For examination with high power a rectangular block of agar about $3 \times 6 \mathrm{~mm}$. was cut, the surrounding agar pulled away and the block raised without distortion by means of a metal lifter (Fig. 1). With the block steadied against

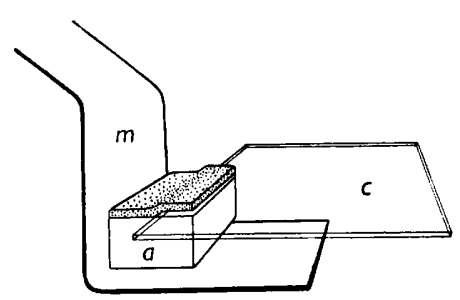

Fig. 1. Technique for separating the agar layers. The full-thickness agar block $a$ is steadied in the shoulder of the bent metal strip $m$, while a cover-glass $c$ is eased between the foundation and seeded layers. the shoulder of the lifter, a cover-glass was gently eased between the two agar layers and the upper layer removed. If the plate has been sufficiently dried this manœuvre presents little difficulty.

As dehydration with $90 \%$ ethanol at this stage makes the individual colonies slightly more distinct, a few drops are run on to the cover-glass, which is gently rocked to ensure that the agar slip is floating freely in the liquid. This is important as the preparation may be too thick for reagents to diffuse freely through from one side alone.

The slip was washed in water, and floated back on to the cover-glass for staining. Methylene blue stains the cocci a bright sky-blue, and the agar purple. With Gram's method decolorization takes much longer than with a simple smear of bacteria, but contrast is good, as agar is coloured pink less intensely than Gram-negative cells when using diluted carbol-fuchsin as a counter-stain.

The slip may be floated on to a slide, and a cover-glass sealed over it with paraffin wax. If desired, a normal blood film may previously be stained on the cover-glass, and mounted film-side under, to provide an approximate measuring gauge for colonial diameters in different parts of the preparation.

Inoculum density. The most satisfactory preparations were obtained by an inoculum giving an even growth of minute, just-discrete colonies. With penicillinase-producers, the inoculum should achieve this density without diminishing the diameter of the inhibition zone by penicillin destruction (cf. the fluid culture work of Barber, 1947 $a, b$ ). The highest tolerated density was determined as follows. When pipette-pen streaks (Ingram, 1951) of graded dilutions of a broth culture were sown at right-angles to a linear penicillin source, at dilutions less than $1 / 100$, the concentration of organisms was inversely related to the distance cleared, but at $\mathbf{1} / \mathbf{1 0 0}$ or higher dilutions (to $1 / 10,000$ ) there was no further increase in cleared distance. The $1 / 100$ dilution of inoculum was therefore used with both the sensitive and the resistant strains, as this dilution satisfactorily fulfilled both criteria. 
Organisms used. (1) The 'Oxford' strain, N.C.T.C. No. 6571, supplied in the dry state from original sources by Dr N. G. Heatley, was the sensitive organism. A pipette-pen streak of a 1/100 broth dilution of an overnight broth culture grew on penicillin agar containing $0.001 \mathrm{unit} / \mathrm{ml}$., but not on $0.01 \mathrm{unit} / \mathrm{ml}$. This technique was designed to test cell sensitivity (Luria, 1946). With a very dense inoculum (produced by centrifuging $2-3 \mathrm{ml}$. of the same broth-culture, and suspending the organisms in 2-3 drops of liquid), a few colonies (most of which appeared to be the minute ' $G$ ' forms) grew on the higher concentration, but none on ten times that concentration. Tested by $\mathrm{Dr}$ A. W. H. Foxell by Kirby's $(1945 b)$ method, it produced no penicillinase.

(2) The resistant strain used was one isolated by Martin \& Whitehead (1949) from human skin, and was known as 'Lumb $\mathrm{C}_{1}$ '. The biochemical reactions were the same as those of the sensitive strain, except that acid was formed rather more slowly in mannitol and milk. Dr V. D. Allison determined the phage type of the resistant strain to be $6 / 47 \mathrm{C}$ (weak reaction); he supplied the information that the Oxford ' $\mathrm{H}$ ' strain did not type with the usual phages, so comparison between the two strains was not possible. A light inoculum produced growth on penicillin agar containing 0.01 but not $0.1 \mathrm{unit} / \mathrm{ml}$, a heavy inoculum growing on 10 but not on 100 units $/ \mathrm{ml}$. When growth occurred it was luxuriant and normal; small-colony forms were not observed. Dr Foxell showed that penicillinase was formed by this resistant strain.

Penicillin. Several different batches of penicillin were used; both organisms were exposed to each sample. No differences were detected in the behaviour of either organism to any batch; in particular, lysis of the sensitive organism was similar with each sample (see Steinmann \& Nicole, 1945).

\section{RESULTS}

The appearances at zone edges formed by the sensitive organism are shown in Pl. 1, figs. 1-3, and by the resistant organism in Pl. 2, figs. 4-6. The thickness of the stripped preparations makes them difficult to photograph and pen-drawings give a better representation of observed phenomena. The only artistic liberty consciously taken has been to represent several focal planes in the same drawing, omitting one set of details in places in order to demonstrate others. Micro-measuring apparatus was not available, but normal human red blood cells were included in the high-magnification drawings to the same scale.

\section{Normal growth}

The surface growth of the sensitive organism occurred in small, widely separated patches, and was never more than one cell thick. With the resistant strain surface-growth was free and continuous, and in places several cells thick (cf. PI. 1, fig. 2 and Pl. 2, fig. 6, upper right-hand corner). The deep growth of the sensitive strain was composed of a mixture of easily distinguished small and large colonies (Pl. 1, fig. 2, C and D), whereas the resistant strain did not show this difference (Pl. 2, fig. 6, lower right-hand corner). 


\section{Effects due to penicillin}

Penicillin affected both the macroscopic appearances of the zone edge, and the microscopic appearance of the cells and colonies.

\section{Macroscopic appearances of the zone edge}

(a) With the sensitive organism the appearance and structure of the zone edge was the same with all sizes of zone tested, except with certain very narrow zones. Typically, three zones could be distinguished. The innermost zone, A (Pl. 1, fig. 1), was only visible with the hand-lens, and had a sharp and regular boundary with the middle zone $B$, visible to the naked eye (one of the many types of 'ghost-zone' which may be encountered in assay plates). The third zone, $\mathrm{C}$, had a clear and regular margin at its common boundary with zone $\mathbf{B}$ but its outer limit was vague and ill-defined. The B/C boundary is of course that usually measured in assay work.

(b) With the resistant organism the appearance of the clear-zone edge was entirely different in narrow and wide zones. In narrow zones about $3 \mathrm{~mm}$. wide the edge was indistinct and quite unsuitable for accurate measurement. Many scattered colonies (some much larger than in the normal growth) were found within the clear area (Pl. 2, figs. 4 (i) and 5). No sharp subdivision of the edge into zones was possible, but the areas of scattered colonies were termed 'scatter zones' to distinguish them from the differently formed 'ghost zones' of the sensitive organism. In large zones, $10 \mathrm{~mm}$. or more in width, the edge was sharp and sufficiently well demarcated to be measured to within $0 \cdot 1 \mathrm{~mm}$. with accuracy, but it was finely and irregularly crenated, so that measurement to this degree of accuracy would not be useful in assay work. The growth in this marginal region was denser than the normal, but was only poorly differentiated from it (Pl. 2, fig. 4 (ii)). With zones of width about 5-8 mm., a transitional form of zone-edge was seen.

\section{Microscopic effects on cell and colony morphology}

(a) With the sensitive organism there was a characteristic cell and colonial morphology in each of the three zones described above (Pl. 1, figs. 2 and 3). In the innermost zone superficial and deep growth was present in about equal amounts, the deep colonies being widely spaced and consisting of few cells only, usually not more than about twenty. The cells were highly pleomorphic and mainly Gram-negative. There were two characteristic abnormalities : (i) groups of two, three or sometimes more enormously swollen and Gram-negative; (ii) cruciate groups of four diplococcal pairs of cells, the inner member of each pair being smaller and sometimes more deeply staining than the outer. Cell debris was found in this region and regarded as evidence of lysis.

In zone $\mathbf{B}$ the colonies were much larger, but still smaller and more translucent than normal. There was no differentiation into large and small colonies. Individual cells were mostly Gram-negative and showed many bizarre features. Nearly all the cells occurred in pairs; in many cases the members were unequal in size and sometimes bizarre.

In zone $\mathrm{C}$ the colonies were rather larger and more opaque than normal, with 
a more irregular surface, which may have been due to an increase in the cell diameter, rather than to an increase of the number of cells. Individual cells were uniformly and weakly Gram-positive, and were almost all in pairs of equal, hemispherical members. In this area the plaques of superficial growth were very much larger than normal, but not more than one cell thick.

(b) With the resistant organism the outstanding and constant feature was the complete absence of any sign of either lysis or of distortion of individual cells. Cells either grew normally in low concentrations of penicillin, or did not grow at all in higher concentrations. Two other observations are also of interest. First, the colonies nearer the penicillin source tended to be larger than normal. In low penicillin concentrations these large colonies were found in the 'scatter zone', but in high concentrations they were confined to a much narrower marginal region just outside the crenated edge of the superficial growth which extended slightly nearer to the penicillin source than did the deep colonies (Pl. 2, figs. 4-6).

Secondly, within the clear area of small and large zones alike there was an even scatter of cells, mostly in ones and twos, normal in appearance and staining reaction, which were thought to be the cells of the original inoculum. This was not found in the preparations of the sensitive strain.

\section{DISCUSSION}

A sensitive and a resistant strain of Staph. aureus have proved to differ in their reactions on penicillin assay plates. A less elaborate study (Ingram, 1951) of twenty-nine strains suggested that these differences are characteristic of the two kinds of staphylococcus, in agreement with the findings of other workers. If these results are confirmed on a larger scale, it would seem that the sensitive kind shows a higher cell sensitivity to penicillin, does not produce penicillinase, and undergoes distortion and lysis when destroyed by this substance, whereas the resistant kind shows a lower cell sensitivity, produces penicillinase, and does not undergo distortion or lysis. Thus, in choosing a test strain for plate assay work, the following considerations may be applied: a sensitive strain will allow relatively low concentrations of penicillin to be assayed, but will carry the disadvantage of an unpredictable number of 'ghost zones' which may be formed by distortion, lysis and, perhaps, secondary growth (Vesterdal); on the other hand, if higher concentrations of penicillin are available for assay, a resistant organism will probably give sharply defined but crenated zone edges with zones above a certain diameter. Below that diameter a 'scatter zone' will be present, formed presumably from more-resistant variants present in the original culture. Comparisons (Ingram, Foxell \& Armitage, unpublished) of the dose-response curves obtained in plate assays made with the sensitive and resistant strains used here, have shown the form of the curves to be similar, despite the production of penicillinase by one strain.

One feature common to the zone edges of both strains was the dense region outside the clear area, fading insensibly into normal growth. 'Secondary zones of stimulation' seen on assay plates with the standard American assay strain of Staph. aureus, FDA 209, were referred to by Cholden (1944), perhaps with this effect in mind. Mitchison \& Spicer (1949) observed that colonies were equally 
numerous in the similar dense zone in a streptomycin assay, and believed that the increased density could not be explained by 'considerations of living space' alone. Dufrenoy and Pratt (Pratt \& Dufrenoy, 1947; Dufrenoy \& Pratt, 1947, 1948) described this dense area explicitly, and after a number of physical and chemical investigations showing cell metabolism to be normal in this region, concluded that the denser growth was due to a stimulation by subtoxic concentrations of penicillin. This interpretation is perhaps also supported by the evidence of Miller, Green \& Kitchen (1945) that staphylococcal populations may increase to higher densities in the presence of subtoxic penicillin concentrations than in control cultures. Such an hypothesis of 'drug stimulation' requires further confirmation, and the dense zone may be more simply explained as the result of the marginal position of the cells concerned. Here they may derive nutriment from a wider field than is available to cells entirely surrounded by others. At the margins of any continuous expanse of bacterial growth dense zones may be seen, formed no doubt in this way, for Mayr-Harting (1947) has shown that, within wide limits, the number of bacterial divisions that can occur on a given area of nutrient surface depends upon available pabulum, and is independent of the size and proximity of individual colonies. Without information on cell distributions the argument of Mitchison \& Spicer is unconvincing.

\section{REFERENCES}

Abraham, E. P., Chain, E., Fletcher, C. M., Gardner, A. D., Heatley, N. G., Jennings, M. A. \& Florey, H. W. (1941). Further observations of penicillin. Lancet, ii, 177.

Barber, M. (1947a). Staphylococcal infection due to penicillin-resistant strains. Brit. med. J. ii, 863.

Barber, M. (1947b). Coagulase-positive staphylococci resistant to penicillin. J. Path. Bact. 59, 373.

Chain, E., Duthie, E. S. \& Callow, D. (1945). Bactericidal and bacteriolytic action of penicillin on the staphylococcus. Lancet, i, 652.

Chapman, G. H. (1945). The significance of sodium chloride in studies of staphylococci. J. Bact. 50, 201.

Cholden, L. S. (1944). A simplified technique for the agar cup assay of penicillin. J. Bact. 47, 402.

Dufrenoy, J. \& Pratt, R. (1947). Cytochemical mechanisms of penicillin action. J. Bact. 53, 657.

Dufrenoy, J. \& Pratt, R. (1948). Evidence for an electrostatic field on penicillin assay plates. Nature, Lond., $161,849$.

Duguid, J. P. (1946). The sensitivity of bacteria to the action of penicillin. Edinb. med. J., 53, 401.

Eriksen, K. R. (1946). Some studies on the lytic action of penicillin on staphylococci and pneumococci. Acta path. microbiol. scand. 23, 221.

Foster, J. W. \& WoodrufF, H. B. (1943). Improvements in the cup assay for penicillin. $J$. biol. Chem. 148, 723 .

Gardner, A. D. (1940). Morphological effects of penicillin on bacteria. Nature, Lond., 146, 837.

Gardner, A. D. (1945). Microscopical effect of penicillin on spores and vegetative cells of bacilli. Lancet, i, 658 .

Ingram, G. I. C. (1951). Classification of staphylococci by penicillin lysis. J. gen. Microbiol. 5, 30. 
Journal of General Microbiology, Vol. 5, No. 1

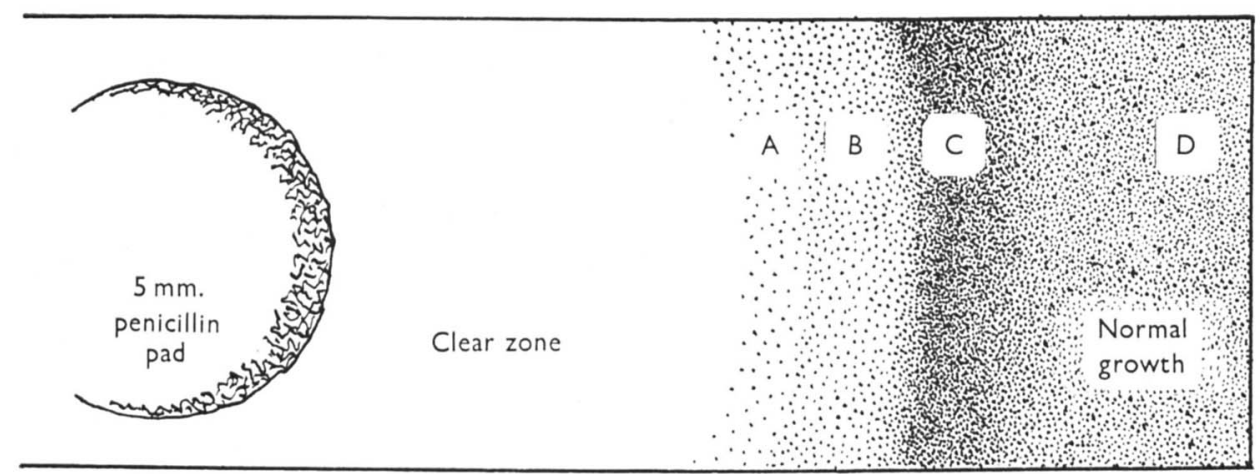

Fig. 1

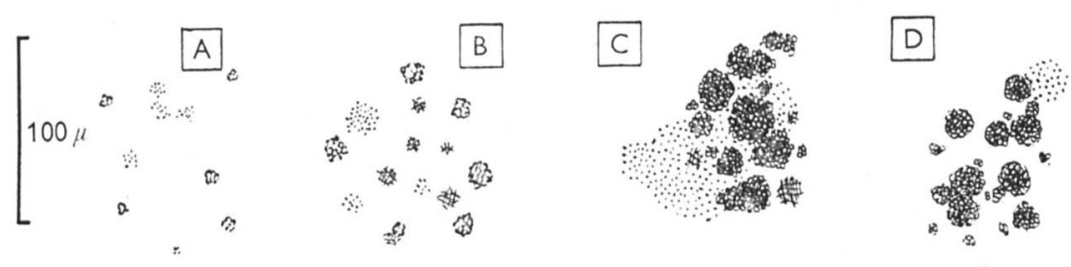

Fig. 2

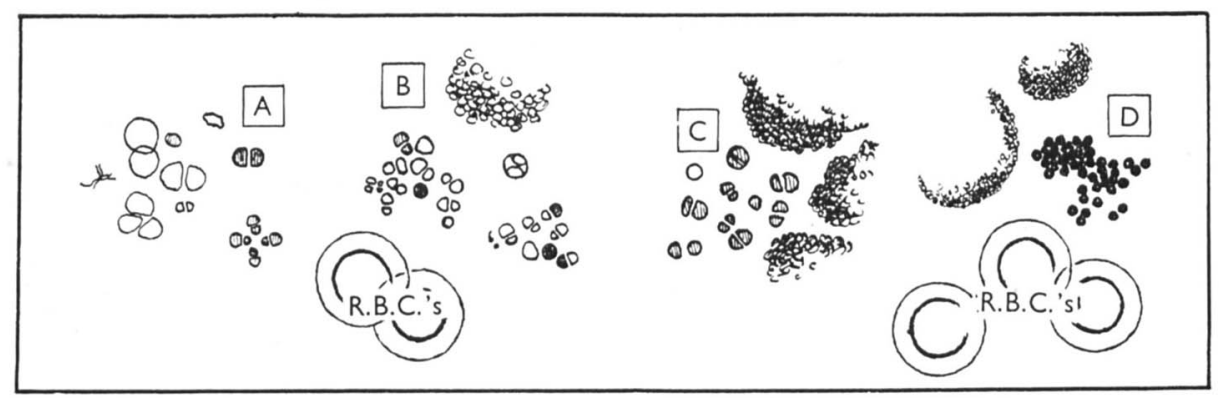

Fig. 3

G. I. C. Ingram-Zones on penicillin assay plates. Plate 1 
Journal of General Microbiology, Vol. 5, No. 1

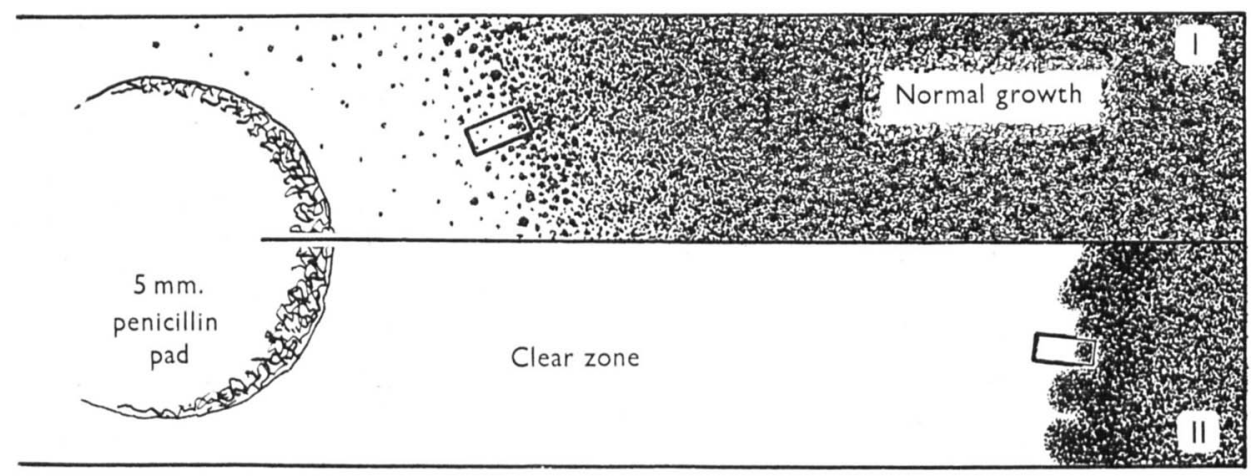

Fig. 4

Distance from edge of pad c. $3 \mathrm{~mm}$.

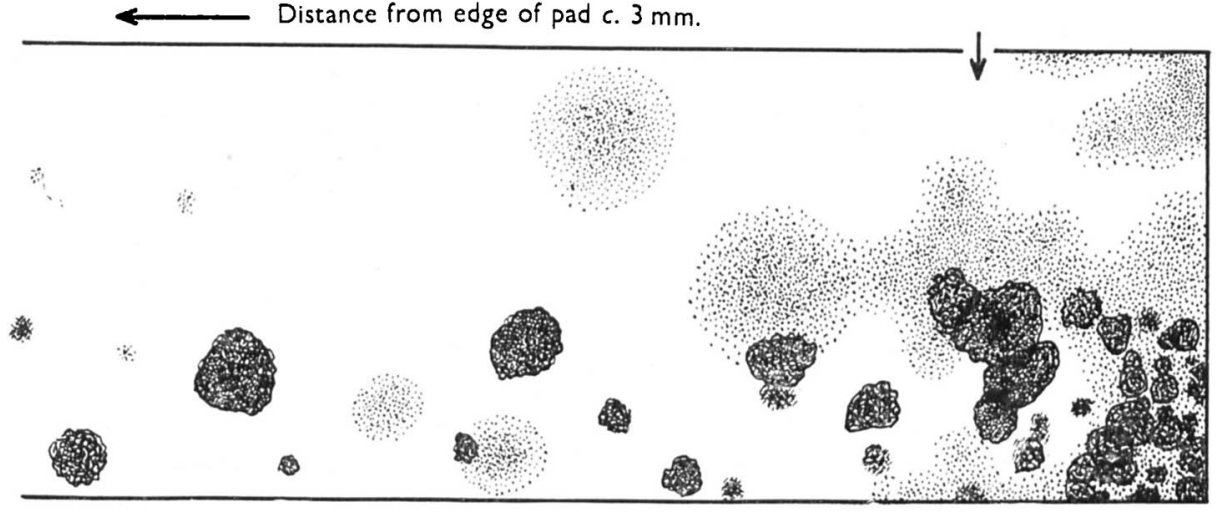

Fig. 5

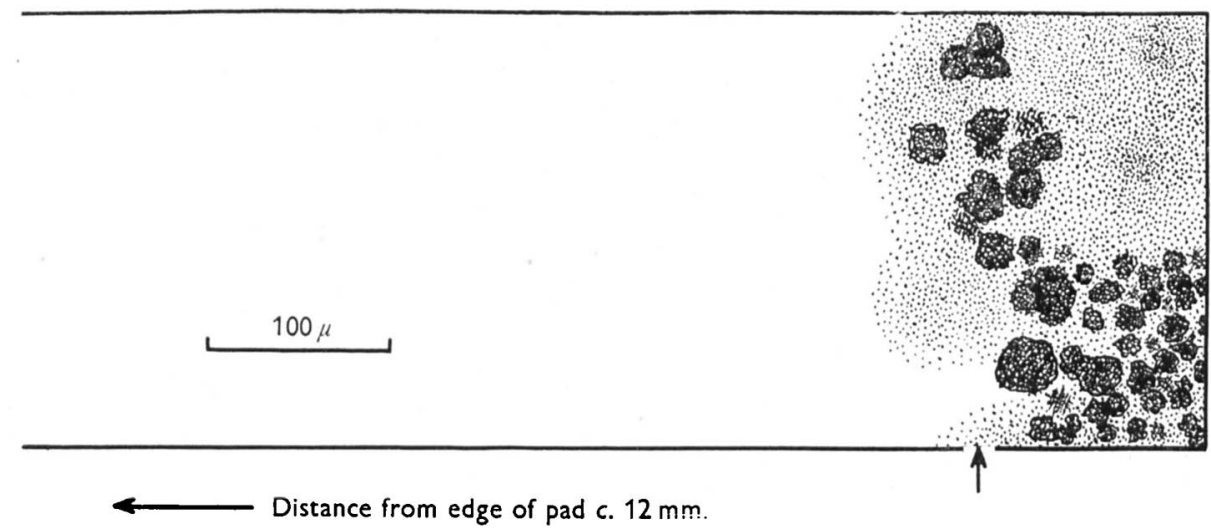

Fig. 6

G. I. C. Ingram-Zones on penicilitin assay plates. Plate 2 
KIRBY, W. M. M. (1945a). Bacteriostatic and lytic actions of penicillin on sensitive and resistant staphylococci. J. clin. Invest. 24, 165.

KIRBY, W. M. M. (1945b). Properties of a penicillin inactivator extracted from penicillin-resistant staphylococci. J. clin. Invest. 24, 170.

KNox, R. (1945). Effect of penicillin on cultures in liquid and solid media. Lancet, i, 559.

LurIa, S. E. (1946). A test for penicillin sensitivity and resistance in staphylococcus. Proc. Soc. exp. Biol., N.Y., 61, 46.

Martin, T. D. M. \& Whitehead, J. E. M. (1949). Carriage of penicillin-resistant Staph. pyogenes in healthy adults. Brit. med. J. i, 173.

Mayr-Harting, A. (1947). The growth of bacterial colonies and their viable population. J. Hyg., Camb., 45, 19.

Miller, W. S., Green, C. A. \& Kitchen, H. (1945). Biphasic action of penicillin and other sulphonamide similarity. Nature, Lond., 155, 210.

Mitchison, D. A. \& Spicer, C. C. (1949). A method of estimating streptomycin in serum and other body fluids by diffusion through agar enclosed in glass tubes. J. gen. Microbiol. 3. 184.

Pratt, R. \& Dufrenoy, J. (1947). Cytochemical mechanisms of penicillin action. II. Changes in reactions of Staphylococcus aureus to vital dyes. J. Bact. 54, 127.

Pulvertaft, R. J. V., Greening, J. R. \& Haynes, J. A. (1947). Liesegang rings and antiseptics. J. Path. Bact. 59, 293.

Steinmann, J. \& Nicole, A. (1945). Quelques correlations entre la penicilline et le bacteriophage. Schweiz. Z. Path. Bakt. 8, 453.

Todd, E. W. (1945). Bacteriolytic action of penicillin. Lancet, i, 74.

Vesterdal, J. (1946). The agar cup method for the estimation of penicillin. Acta pharmacol. toxicol. 2, 9.

\section{EXPLANATION OF PLATES}

Plate 1

Figs. 1-3. The sensitive strain of Staph. aureus.

Fig. 1. General appearances at the zone-edge formed round a disk reservoir on a surface pre-seeded plate. Lettered areas shown enlarged below. Unstained, fresh preparation: hand-lens examination.

Fig. 2. Colonial appearances in the lettered areas of fig. 1. Relative extent of plaques of superficial growth shown by stippling; deep colonies drawn dark. Unstained, fresh preparation: $\frac{2}{3}$ in. objective.

Fig. 3. Cell morphology of the same areas. Colony profiles intended only to show general intensity of staining. Gram-stained preparation (technique described); $\frac{-\frac{1}{12}}{\text { in. oil }}$ immersion. Red blood cells included for scale.

Plate 2

Figs. 4-6. The resistant strain of Staph. aureus.

Fig. 4. General appearances at the zone-edge formed on a surface pre-seeded plate round disk reservoirs carrying low (i) and high (ii) penicillin concentrations. Enclosed areas shown enlarged below. Unstained, fresh preparations, comparable with fig. 1. Hand-lens examination.

Fig. 5. Colonial appearances in the area enclosed in fig. 4 (i). Deep colonies (drawn dark) omitted from the upper half of the drawing to show superficial growth alone (stippled). Unstained, fresh preparation, comparable with fig. $2: \frac{2}{3}$ in. objective.

Fig. 6. Colonial appearances in the area enclosed in fig. 4 (ii). Deep colonies omitted from the top right-hand part of the picture to show how the superficial growth was continuous and in places heaped up. Technique and scale as in fig. 5. 